\title{
Studies on the in Vitro Dissolution of Insoluble Volatile Drug from Su-Anxin Nasal Inhalant and Its Correlation on the Nose Steady Self-Controllable Expiration and Inspiration at Night
}

\author{
Xiaodong $\mathrm{Li}^{1,2}$, Guoxiang $\mathrm{Xie}^{2}$, Suyun $\mathrm{Li}^{1,3}$, Chao $\mathrm{Hou}^{1,3}$ \\ ${ }^{1}$ College of Pharmacy, Fujian University of Traditional Chinese Medicine, Fuzhou, China; ${ }^{2}$ Department of Nutrition, University of \\ North Carolina at Greensboro, North Carolina Research Campus, Kannapolis, USA; ${ }^{3}$ Nasal preparation institute of traditional Chi- \\ nese medicine of Fujian province, College of Pharmacy, Fujian University of Traditional Chinese Medicine, Fuzhou, China. \\ Email:1xdtcm@163.com
}

Received February $1^{\text {st }}, 2011$; revised March $2^{\text {nd }}, 2011$; accepted March $12^{\text {th }}, 2011$.

\begin{abstract}
In the paper, the in vitro dissolution of borneol in 12 hours from 6 batches of optimized inhalant samples were investigated. As a new dosage form, the in vitro release apparatus of nasal inhalant was invented and a pushing bump was used according to the simulation of the nose expiration and inspiration. Based on the data of $r^{2}$ in the profile and similar factor $f_{2}$ from 6 linear release tendencies, a good controlled release and a zero order tendency were observed. It can be suggested that there is a good correlation between the in vitro controlled release and the nose steady self-controllable expiration and inspiration, which will contribute to the trend of insoluble volatile drug controlled release and the effect of quick absorption in nasal pulmonary delivery to cure severe or acute cardiovascular or lung diseases at patients' sleeping, such as angina or breathing obstruction. Also, it was concluded that the prescription composed of insoluble volatile drugs can be prepared to be nasal inhalant from which drugs can be absorbed through nose steady self-controllable inspiration to the lung then into the blood and have a great effectiveness improvement of bioavailability at night timing drug delivery system.
\end{abstract}

Keywords: Su-Anxin Nasal Inhalants, Insoluble Volatile Drugs, Timing Drug Delivery System, In Vitro Dissolution, Controlled Release

\section{Introduction}

Aromatherapy, a practice of therapeutic use of essential plant-based oils or other volatile materials for relieving anxieties and stresses, improving body immunity and curing some uncertain diseases, has played a great role in the care of our health and many products of natural essential oil, such as cosmetics, are gaining a pretty good sale every year. In China, the herbs with essential oils have been used as traditional Chinese medicine to cure different kinds of diseases for several thousand years. In recent literatures, quite a lot of researches were reported for aromatherapy to have an good effect on Alzheimer's disease, anxiety symptoms, stress, supportive care of cancer patients, hypertension, cognition and mood modula- tion, psychiatric disorders, inhalational anaesthetic agents, cardiovascular disease and so on [1-11].

In this paper a new use mode and dosage form, named Su-anxin nasal inhalant, was mainly introduced to cure cardiovascular or pulmonary disease in patients' sleeping. As a kind of aromatherapy, this nasal inhalant not only gave the scientific proofs at its device and in vitro release test, but also brought a product for healthcare with good convenience, low cost, steady dose and high bioavailability.

Su-anxin nasal inhalant was defined as an inhaled preparation which is composed of a kind of equipment with two sides, one stuck to the middle part, called philtrum, under the nose with the help of some stickers, the 
other holding the insoluble volatile drug that are exposed at atmosphere after discarding the cover, and will be absorbed through nose inspiration into the lung to cure the diseases in a systemic or local form [12] (Figure 1). It consists of some volatile components, such as styrax and borneol, having an excellent therapy for angina, chest distress and breathing obstruction especially suddenly happened at night when a middle or old age patient is in sleep. Compared with such oral cardiovascular preparation as Suhexiang pill, Shexiangbaoxin pill and compound dropping pill of Danshen root in China, Su-anxin nasal inhalant has good merits of quick absorption, high bioavailability and first metabolism without passing through the liver. It will be great helpful because it can avoid the patient's sudden death induced from these diseases through a good controlled inhalation at the whole night. Referred to plenty of literatures on inhaled preparation reported [13-21], it is a good choice for insoluble volatile drug to be nasal inhalant to cure such diseases, especially essential oils.

Using the method of gas chromatography, the tests about the in vitro accumulative release percentage of

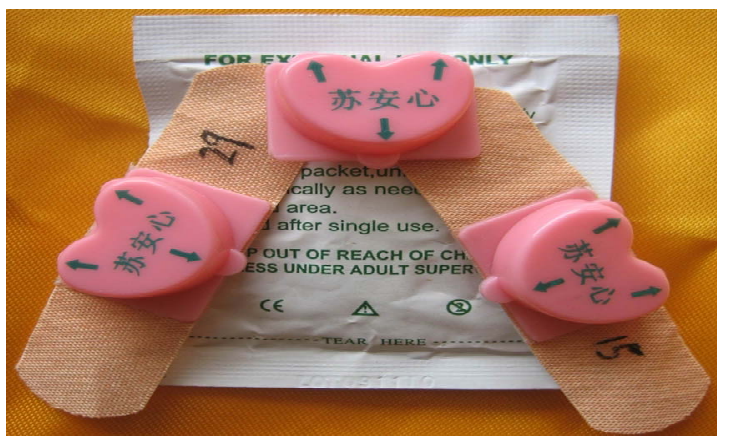

(a)

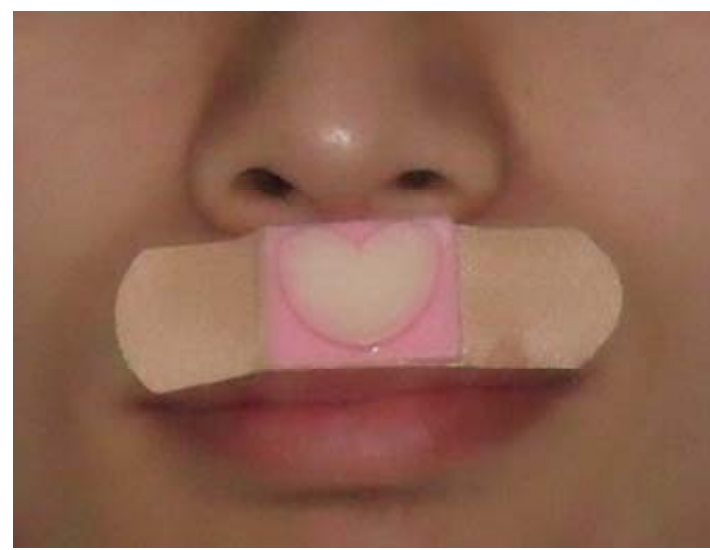

(b)

Figure 1. Real product profile of Su-anxin nasal inhalant. ((a): the whole view of the product; (b): simulated use under the nose after discarding the cover).

borneol in nasal inhalant were done under the simulation of nose expiration and inspiration, which will contribute to the research and development of nasal inhalants for good nasal inhalants and improving their bioavailability at night timing drug delivery system.

\section{Materials and Methods}

\subsection{Materials and Agents}

Six batches of optimized Su-anxin nasal inhalants were made by our laboratory colleagues, each piece $0.45 \mathrm{~g}$. FA2004N type electronic balance (Shanghai Jing-mi scientific instrument Ltd. Co., China), KQ-500E type ultrasonic cleaning(Ultrasonic instrument Ltd. Co. of Kunshan, China), standard borneol (National institute for the control of pharmaceutical and biological products, batch number 110743-200504), stearic acid (Tianjin Zhi-yuan chemical industry Ltd. Co.,China), hydroxypropylmethylcellulose(HPMC, K 15 M, Colorcon Co., U.K.), Starch (Tianjin Fu-chen chemical reagent company, China), sodium lauryl sulfate (SLS, Tianjin Bo-di chemical industry Ltd. Co.,China), sodium carboxymethycellulose (CMC$\mathrm{Na}$, Tianjin Da-mao chemical reagent company, China) and trolamine (Shanghai Lian-shi chemical reagent Ltd. Co., China) were needed. All other reagents used were analytical grade.

\subsection{The Preparation of Optimized Su-Anxin Nasal Inhalants}

After $0.075 \mathrm{~g}$ stearic acid, $0.075 \mathrm{~g}$ trolamine and $0.15 \mathrm{~g}$ petrolatum liquidum were weighed, mixed and melted, $1.5 \mathrm{~g}$ HPMC was added to $5 \mathrm{ml}$ water to form gel and mixed uniformly. Then, prescribed borneol, styrax and other drugs were weighed and dissolved with $75 \%$ alcohol to form drug solution. The drug solution was put into the above mixture and mixed. Then, $3 \mathrm{~g}$ starch and $2 \mathrm{~g}$ CMC-Na were added to them again and mixed uniformly to form Su-anxin nasal inhalant finally.

\subsection{The Chromatographic Condition of GC}

$6890 \mathrm{~N}$ type gas chromatography and soft work station were used (Agilent company, USA). The method includes DB-5 MS type capillary column $(30 \mathrm{~m} \times 0.25 \mathrm{~mm}$ i.d $\times$ $0.25 \mu \mathrm{m})$, a column temperature of $80^{\circ} \mathrm{C} \stackrel{5^{\circ} \mathrm{C} / \min }{\longrightarrow} 220^{\circ} \mathrm{C}$ (keeping $25 \mathrm{~min}$ at $220^{\circ} \mathrm{C}$ ), a $\mathrm{He}$ carrier gas, a flowing rate of $1 \mathrm{~mL} / \mathrm{min}$, a temperature of sampling entrance at $250^{\circ} \mathrm{C}$, a diffluent rate of $10: 1$ and a FID detector with a temperature $280^{\circ} \mathrm{C}$. The sampling size was $1 \mu \mathrm{L}$.

\subsection{The Preparation of Samples}

$0.45 \mathrm{~g}$ sample was weighed precisely and made it a thin layer. Then, it was put into $45 \mathrm{ml}$ dehydrated alcohol in the flask with a glass stopper and shaken for $5 \mathrm{~min}$. After 
$24 \mathrm{~h}$ in a tight state, the solution was ultrasounded $10 \mathrm{~min}$ and filtered into $50 \mathrm{ml}$ measuring flask. Moreover, moderate dehydrated alcohol was added to wash the flask with a glass stopper and filtered into measuring flask again. After metered with dehydrated alcohol, the sample solution was prepared. The total six batches were done.

\subsection{The Preparation of Standard Solution}

Standard borneol $25 \mathrm{mg}$ was weighed precisely and put it into $25 \mathrm{~mL}$ measuring flask. After dissolved and metered by dehydrated alcohol, the standard solution at real concentration $1.004 \mathrm{mg} / \mathrm{mL}$ was obtained.

\subsection{The Preparation of Negative Solution}

Prescribed styrax and other drugs except borneol were weighed and negative product was prepared on the basis of the item 2.2. Then, according to the item 2.4, the negative solution was obtained.

\subsection{Dissolution Studies}

Borneol releases from the different 6 batches inhalants were determined using self-made dissolution apparatus (Figure 2) designed for simulation of our nose expiration and inspiration with $50 \mathrm{ml}$ of dehydrated alcohol as dissolution medium. The temperature of the dissolution medium was maintained at room temperature. $20 \mathrm{ml}$ dehydrated alcohol was added into conical flask and the inhalant sample was put in the intubation tube with the drug surface upwards. One point of intubation tube was set beneath the liquid of dehydrated alcohol in the conical flask and, the other was blocked by a glass stopper. There is an inlet beside the intubation tube near to the stopper,

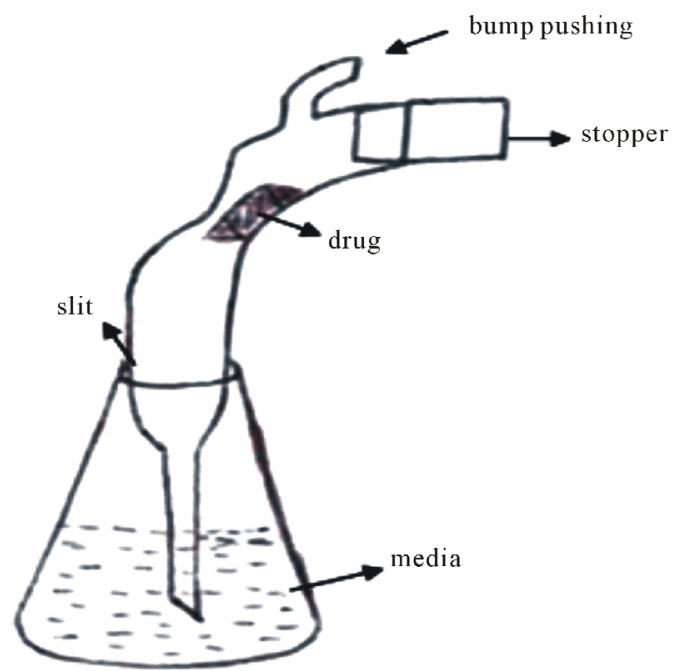

Figure 2. the test apparatus of the in vitro release for inhalants.

through which the mild wind was pushed into the tube by a pushing bump and the gas from insoluble volatile drug was taken into the liquid and dissolved in the dehydrated alcohol with the wind while other gases from the atmosphere were sent out to atmosphere. When the release time came, the push bump was turned off and the conical flask was taken down. Meanwhile, a new conical flask with $20 \mathrm{ml}$ dehydrated alcohol in it was connected to the intubation tube quickly to assure the next continual test. Then, previous about $20 \mathrm{ml}$ dehydrated alcohol was put into measuring flask and metered. Samples of $20 \mathrm{ml}$ were withdrawn at 0.5, 1.0, 2.0, 4.0, 6.0, 8.0 and 12.0 hour and replaced $20.0 \mathrm{ml}$ fresh dissolution media every scheduled time.Analysis of dissolved borneol in the dehydrated alcohol solution was conducted by GC method. All experiments were performed in triplicate.

\section{Results and Discussion}

\subsection{The Calibration Curve of Borneol}

To determine the linearity of the GC detector response, calibration standard solutions of borneol at the concentration of 20.08, 40.16, 60.24, 80.32 and $100.4 \mu \mathrm{g} / \mathrm{mL}$ were prepared on the basis of item 2.5. Linear correlation was obtained between peak areas versus concentration of borneol. Each measurement represented the average of three replicates. The regression equation was $\mathrm{A}=4.8582 \mathrm{C}+$ $14.809(\mathrm{r}=0.9998)$, which indicates a good linear relation between peaks and borneol concentration at the range of $20.08 \sim 100.4 \mu \mathrm{g} / \mathrm{ml}$. Related spectrum can be seen at Figure 3.

\subsection{Accuracy, Reproducibility, Repeatability and Recovery}

Accuracy was performed by running the borneol standard solution of $60.24 \mu \mathrm{g} / \mathrm{ml}$ for 5 continual times under the same conditions. Reproducibility was evaluated by analyzing one sample after diluted 10 times at interval 8 hours. Repeatability was performed by running 5 pieces of samples after diluted 10 times under the same conditions. Recoveries varied between $99.0 \%$ and $104.4 \%$ with RSD $1.96 \%$. The results obtained shows that the method is very accurate and precise, which were presented in Tables 1-4.

\subsection{Determination of Borneol in Sample}

The total 6 batches samples were analyzed by GC method and average determination were $8.26 \%, 8.96 \%, 8.21 \%$, $8.37 \%, 8.50 \%$ and $8.32 \%$ respectively, which could be used as the total quantity of borneol in the test of the in vitro dissolution. 


\subsection{The Release of Borneol from Su-anxin Inhalants}

In order to express the correlation between the controlled release and the nose expiration and inspiration at night, a series of our previous screenings of prescription on $\mathrm{o} / \mathrm{w}$, w/o and other excipients' choice [22] were cancelled and just the in vitro dissolution release behaviors of borneol from 6 batches optimized Su-anxin inhalants were done. The all accumulative releases of borneol from inhalants were controllable, showing a tendency of zero order release (the lest $r^{2}=0.9784$ ). Table 5 and Figure 4 illustrated the data and the profile of the release in 12 hours. However, if the illustration was shown without the point of the time at 0.5 hour, the tendency of zero order release was more obvious than that in 12 hours, which could be seen in Table 6 and Figure 5. From the point at 0.5 hour, the largest release percentage was observed, showing the first large concentration of insoluble volatile drug at the surface of the preparation and a prompt absorption through nasal lung delivery. The phenomenon was interestingly accorded with the previous in vivo test which gave a Cmax of borneol in the blood of rabbits [23].

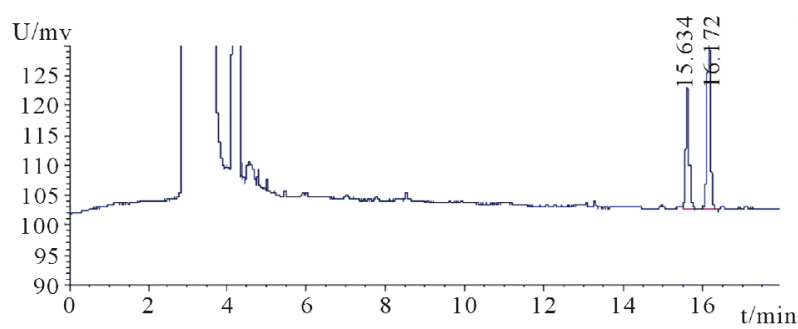

(a)

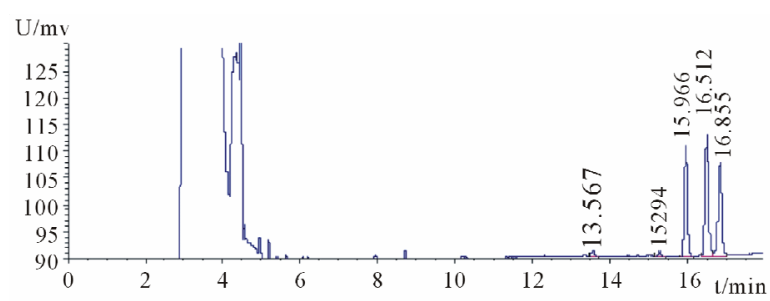

(b)

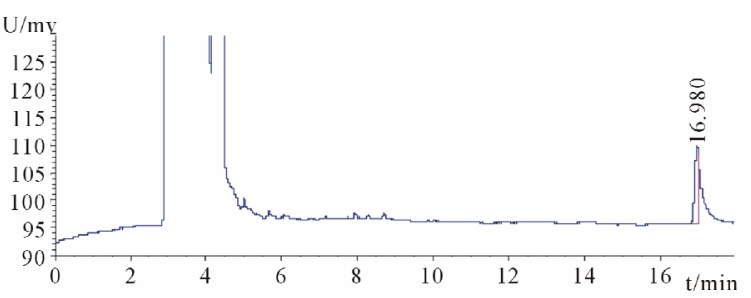

(c)

Figure 3. The GC illustration of borneol in inhalant. ((a) standard solution; (b) sample solution; (c) negative sample solution without borneol).
Table 1. The data on the accuracy trial $(n=5)$.

\begin{tabular}{llllll}
\hline times & 1 & 2 & 3 & 4 & 5 \\
\hline peak area & 295.4 & 298.6 & 292.1 & 297.2 & 294.8 \\
RSD\% & 1.78 & & & & \\
\hline
\end{tabular}

Table 2. The data on the reproducibility trial $(n=6)$.

\begin{tabular}{lllllll}
\hline time $(\mathrm{h})$ & 0.5 & 1 & 2 & 4 & 6 & 8 \\
\hline peak area & 422.0 & 410.1 & 402.6 & 400.0 & 394.2 & 387.1 \\
RSD\% & 3.04 & & & & & \\
\hline
\end{tabular}

Table 3. The data on the repeatability trial $(n=5)$.

\begin{tabular}{llllll}
\hline times & 1 & 2 & 3 & 4 & 5 \\
\hline peak area & 369.1 & 363.0 & 359.1 & 347.8 & 354.3 \\
$R S D \%$ & 2.27 & & & & \\
\hline
\end{tabular}

Table 4. The data on the recovery trial $(n=6)$.

\begin{tabular}{lcccccc}
\hline $\begin{array}{l}\text { samples } \\
(\mathrm{mg})\end{array}$ & $\begin{array}{l}\text { borneol in } \\
\text { samples } \\
(\mathrm{mg})\end{array}$ & $\begin{array}{l}\text { added } \\
\text { standard } \\
(\mathrm{mg})\end{array}$ & $\begin{array}{l}\text { Theoretical } \\
\text { borneol } \\
(\mathrm{mg})\end{array}$ & $\begin{array}{l}\text { Measured } \\
\text { borneol } \\
(\mathrm{mg})\end{array}$ & $\begin{array}{l}\text { recovery } \\
(\%)\end{array}$ & $\begin{array}{l}\text { RSD } \\
\%\end{array}$ \\
\hline 223.7 & 19.9 & 20.1 & 40.0 & 39.8 & 99.0 & \\
219.5 & 19.6 & 20.2 & 39.8 & 39.9 & 100.5 & \\
222.9 & 19.9 & 20.2 & 40.1 & 40.5 & 102.0 & 1.96 \\
224.2 & 20.0 & 20.3 & 40.3 & 40.6 & 101.5 & \\
223.1 & 19.9 & 19.9 & 39.8 & 39.7 & 99.5 & \\
218.6 & 19.5 & 20.3 & 39.8 & 40.7 & 104.4 & \\
\hline
\end{tabular}

Table 5. The data of in vitro accumulative release from 6 batches inhalants.

\begin{tabular}{llllllll}
\hline time(h) & 0.5 & 1 & 2 & 4 & 6 & 8 & 12 \\
\hline batch1(\%) & 2.38 & 3.95 & 7.41 & 10.43 & 14.07 & 16.89 & 22.1 \\
batch2(\%) & 2.36 & 3.63 & 5.37 & 8.89 & 12.07 & 14.92 & 19.83 \\
batch3(\%) & 2.03 & 3.36 & 5.22 & 8.89 & 12.05 & 15.01 & 20.04 \\
batch4(\%) & 2.85 & 5.21 & 7.15 & 11.25 & 14.93 & 18.06 & 23.18 \\
batch5(\%) & 2.5 & 4.18 & 6.09 & 9.86 & 12.57 & 15.98 & 20.91 \\
batch6(\%) & 2.67 & 4.52 & 7.15 & 11.14 & 14.14 & 17.09 & 22.65 \\
\hline
\end{tabular}

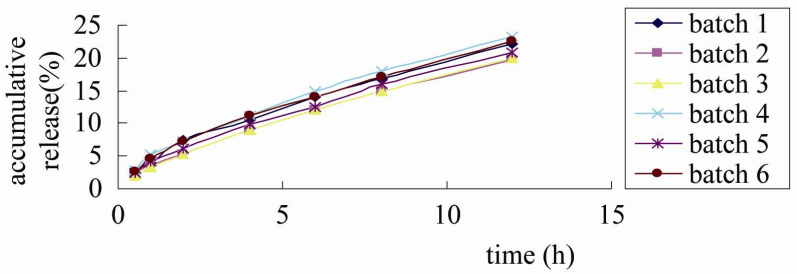

Figure 4. The dissolution release profile of borneol from inhalants in 12 hours. 
Table 6. The linear comparison of $\mathbf{r}^{2}$ between two kind of release profile.

\begin{tabular}{lllllll}
\hline batches & 1 & 2 & 3 & 4 & 5 & 6 \\
\hline $\mathrm{r}^{2}$ including $0.5 \mathrm{~h}$ & 0.9784 & 0.9922 & 0.9914 & 0.9821 & 0.9893 & 0.9832 \\
$\mathrm{r}^{2}$ without $0.5 \mathrm{~h}$ & 0.9836 & 0.9934 & 0.9930 & 0.9890 & 0.9930 & 0.9892 \\
\hline
\end{tabular}

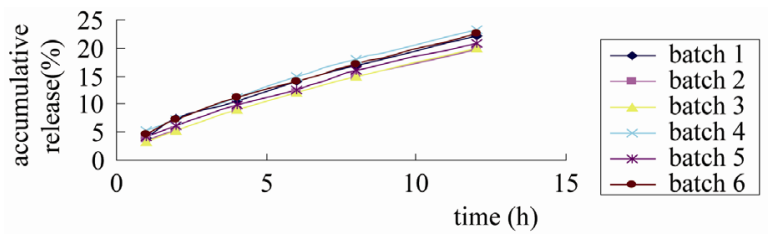

Figure 5. The dissolution release profile of borneol from inhalants in 12 hours without 0.5 hour point.

Also, on the basis of the equation [24] of similar factor of release curve, $f_{2}$ :

$$
f_{2}=50 \times \lg \left\{\left[1+(1 / n) \sum W_{t}\left(R_{t}-T_{t}\right)^{2}\right]^{-1 / 2} \times 100\right\}
$$

where $R_{t}$ is the release of the reference sample at $t$ time, $\mathrm{T}_{\mathrm{t}}$ the release of the test sample at $\mathrm{t}$ time, $\mathrm{n}$ the number of the sampling point and $W_{t}$ weight factor (here $W_{t}=1$ ). After calculation, the $f_{2}$ values were obtained and the smallest was 79.16 compared linear tendency of batch 2 and that of batch 4, the largest was 99.61 between batch 2 and batch 3 , which indicated a good accordance among 6 batches samples.

\subsection{The Elucidation of the Correlation between Release and Nose Expiration and Inspiration}

In the in vitro release at item 2.7, the pushing bump simulated the nose of human being and modulated to a mild fixed pushing rate was used. Though the direction was one way and different from the person's nose with two ways by expiration and inspiration. Since Su-anxin inhalant is used for curing the cardiovascular disease at night when nose has an expiration and inspiration at a steady frequency during someone's sleeping, the total quantity between accumulative release and nose expiration and inspiration was reckoned to the same for some extent. However, because there is a very short stop for nose exchange between expiration and inspiration when a person's sleeping, the total quantity of nose expiration and inspiration will a little larger than that of accumulative release, which can be verified in the near trial. In a word, it is useful and valuable for the practice of the inhalants after the elucidation of the correlation between release and nose expiration and inspiration.

\section{Conclusions and Discussion}

Analysis results indicated that the determination method of borneol is sensitive, accurate, and reduplicate, which can be used to determine borneol from Su-anxin nasal inhalants. Based on this, the 6 batches of sample inhalants showed a good controlled release tendency according to $r^{2}$ in the profile and similar factor $f_{2}$, which explained the excellent preparation of the optimized Suanxin inhalant. As a new dosage form, the in vitro release apparatus was invented and a pushing bump was used after the simulation of the nose expiration and inspiration, which confirmed a good correlation between the in vitro release and the nose expiration and inspiration when used at the possible same condition. It can be suggested that the nose, the same as what the pushing bump did, is very important in bringing the trend of drug controlled release and the effect of quick absorption when used for the patients.

For insoluble volatile drugs, it is appropriate to prepare them for the formulation of nasal inhalant. It will bring a good drug night timing delivery and improved bioavailability when used. It is very interesting that when the patient is inhaling the inhalant to cure certain disease, his or her spouse can also inhale the volatile gas and have a more quiet and better sleep for the fragrant smell of the volatile drug.

In the dissolution test, some amount of volatile drug was possibly lost if dehydrated alcohol could evaporate with the air. Because the boiling point of alcohol is $78^{\circ} \mathrm{C}$ and the test was done at the room temperature, the real lost of borneol was very small. It has little effect on the accumulative release. The test in a low temperature will be done in our next future tests to check the real value of volatile drug. Meanwhile,because the mixture of volatile materials,such as borneol and styrax, had a relatively similar release tendency when released from the excipients of the inhalant, the borneol was selected for the in vitro test model for its simple analytic method.

\section{Acknowledgements}

Be grateful for the support of grants from Chinese Ministry of Education, Fujian Provincial Department of Education, Fujian Provincial Department of Science and Technology, and the Foundation of Integrated Traditional Chinese and Western Medicine from Chen Ke-ji (No. 209064, JA08112, 2005J045 and CKJ2007007).

\section{REFERENCES}

[1] M. Shakeel, A. Trinidade, S. Jehan and K. W. Ah-See, "The Use of Complementary and Alternative Medicine by Patients Attending a General Otolaryngology Clinic: Can We Afford to Ignore It?" American Journal of Otolaryngology, Vol. 31, No. 4, 2010, pp. 252-260. doi:10.1016/j.amjoto.2009.02.016 
[2] K. J. Hunt, H. F. Coelho, B. Wider, R. Perry, S. K. Hung, R. Terry and E. Ernst, "Complementary and Alternative Medicine Use in England: Results from a National Survey," International Journal of Clinical Practice, Vol. 64, No. 11, 2010, pp. 1496-1502. doi:10.1111/j.1742-1241.2010.02484.X

[3] D. Jimbo, Y. Kimura, M. Taniguchi, M. Inoue and K. Urakami, "Effect of Aromatherapy on Patients with Alzheimer's Disease," Psychogeriatrics, Vol. 9, No. 4, 2009, pp. 173-179. doi:10.1111/j.1479-8301.2009.00299.x

[4] L. Weinberg, D. Story, J. Nam and L. Mcnicol, "Pharmacoeconomics of Volatile Inhalational Anaesthetic Agents: An 11-Year Retrospective Analysis," Anaesthesia and intensive care, Vol. 38, No. 5, 2010, pp. 849-854.

[5] D. K. Conn and D. P. Seitz, "Advances in the Treatment of Psychiatric Disorders in Long-Term Care Homes," Current Opinion in Psychiatry, Vol. 23, No. 6, 2010, pp. 516-521. doi:10.1097/YCO.0b013e32833efe56

[6] E. Heuberger and J. Llmberger, "The Influence of Essential Oils on Human Vigilance," Natural Product Communications, Vol. 5, No. 9, 2010, pp. 1441-1446.

[7] L. Moss, M. Rouse, K. A. Wesnes and M. Moss, "Differential Effects of the Aromas of Salvia Species on Memory and Mood," Human Psychopharmacology, Vol. 25, No. 5, 2010, pp. 388-396. doi:10.1002/hup.1129

[8] H. W. Tsang and T. Y. Ho, "A Systematic Review on the Anxiolytic Effects of Aromatherapy on Rodents under Experimentally Induced Anxiety Models," Reviews in the Neurosciences, Vol. 21, No. 2, 2010, pp. 141-152.

[9] M. H. Hur, M. S. Lee, C. Kim and E. Ernst, "Aromatherapy for Treatment of Hypertension: A Systematic Review," Journal of Evaluation in Clinical Practice, Vol. 17, No. 2, 2010, pp. 1365-2753.

[10] J. Tillett and D. Ames, "The Uses of Aromatherapy in Women's Health," Journal of Perinatal \& Neonatal Nursing, Vol. 24, No. 3, 2010, pp. 238-245.

[11] A. Supoken, T. Chaisrisawatsuk and B. Chumworathayi, "Proportion of Gynecologic Cancer Patients Using Complementary and Alternative Medicine," Asian Pacific Journal of Cancer Prevention, Vol. 10, 2009, pp. 779782.

[12] X. D. Li and L. P. Zhang, "The Application Prospect of Nasal Inhalant of Traditional Chinese Medicine," Journal of Fujian College of Traditional Chinese Medicine, Vol. 17, 2007, pp. 56-58.

[13] S. T. Charlton, S. S. Davis and L. Illum, "Evaluation of
Bioadhesive Polymer as Delivery Systems for Nose to Brain Delivery: In Vitro Characterisation Studies," Journal of Controlled Release, Vol. 118, No. 2, 2007, pp. 225-234. doi:10.1016/j.jconrel.2006.12.014

[14] R. Siekmeier and G. Scheuch, "Inhaled Insulin-Does It Become Reality?" Journal of Physiology and Pharmacology, Vol. 59, No. 6, 2008, pp. 81-113.

[15] B. Luppi, F. Bigucci, T. Cerchiara and V. Zecchi, "Chitosan-Based Hydrogels for Nasal Drug Delivery: From Inserts to Nanoparticles," Expert Opinion on Drug Delivery, Vol. 7, No. 7, 2010, pp. 811-828. doi: $10.1517 / 17425247.2010 .495981$

[16] K. W. Spencer, "Isopropyl Alcohol Inhalation as Treatment for Nausea and Vomiting," Plastic Surgical Nurses, Vol. 24, No. 4, 2004, pp. 149- 154.

[17] C. Li, C. S. Gao and X. G. Mei, "The Research Development of Protain/Polypeptide Drugs in Pulmonary Inhaled Preparation," Foreign Medical Sciences Section of Pharmacy, Vol. 34, 2007, pp. 56-58.

[18] J. P. Zhu and R. S. Zhao, "Inhaled Delivery Drugs and Their Clinical Application and Evaluation," Clinical Medication Journal, Vol. 6, 2008, pp. 49-52.

[19] Y. C. Hong and H. W. Huang, "To Cure Chronic Rhinitis Through a Main Drug Delivery: Chinese Medicine Aerosol," Journal of Modern Integrated Medicine, Vol. 11, 2002, p. 1097

[20] Q. L. Yang, "The Treatment of Respiratory Diseases: Chinese Medicine Aerosol," Journal of Liaoning University of Traditional Chinese Medicine, Vol. 10, 2008, pp. 103-104.

[21] L. Illum, "Nasal Drug Delivery_-Possibilities, Problems and Solutions," Journal of Controlled Release, Vol. 87, No. 1-3, 2003, pp. 187-198. doi:10.1016/S0168-3659(02)00363-2

[22] X. D. Li and S. Y. Li, "Studies on Improving Absorption of Insoluble Volatile Drugs from Su-Anxin Nasal Inhalant," The Proceedings of 2010 Second Asia Arden PharmaceuTical Technology Forum, 279.

[23] X. D. Li, Y. Chen and F. H. Wu, "Analysis of in Vitro And in Vivo Drug Components in Rabbits from SuAnxin Nasal Inhalant," Journal of Chengdu Medical College, Vol. 5, No. 2, 2010, pp. 119-121.

[24] P. Costa, "An Alternative Method to the Evaluation of Similarity Factor in Dissolution Testing," International Journal of Pharmaceutics, Vol. 220, No. 2, 2001, pp. 77-83. doi:10.1016/S0378-5173(01)00651-2 